Musées, Patrimoine et Culture scientifiques et techniques

$190 \mid 2020$

juillet-août 2020

\title{
Musées et nouvelles modalités attractives
}

\section{Lorenzo Greppi}

\section{OpenEdition}

Journals

Édition électronique

URL : https://journals.openedition.org/ocim/4047

DOI : $10.4000 /$ ocim.4047

ISSN : 2108-646X

Éditeur

OCIM

Édition imprimée

Date de publication : 1 juillet 2020

Pagination : 24-29

ISSN : 0994-1908

Référence électronique

Lorenzo Greppi, « Musées et nouvelles modalités attractives », La Lettre de l'OCIM [En ligne], 190 | 2020 mis en ligne le 01 juillet 2021, consulté le 17 juillet 2021. URL : http://journals.openedition.org/ocim/ 4047 ; DOI : https://doi.org/10.4000/ocim.4047

Ce document a été généré automatiquement le 17 juillet 2021.

Tous droits réservés 


\section{Musées et nouvelles modalités attractives}

\section{Lorenzo Greppi}

Ces « animaux qui bougent dans l'air » au musée d'Histoire naturelle de Venise.

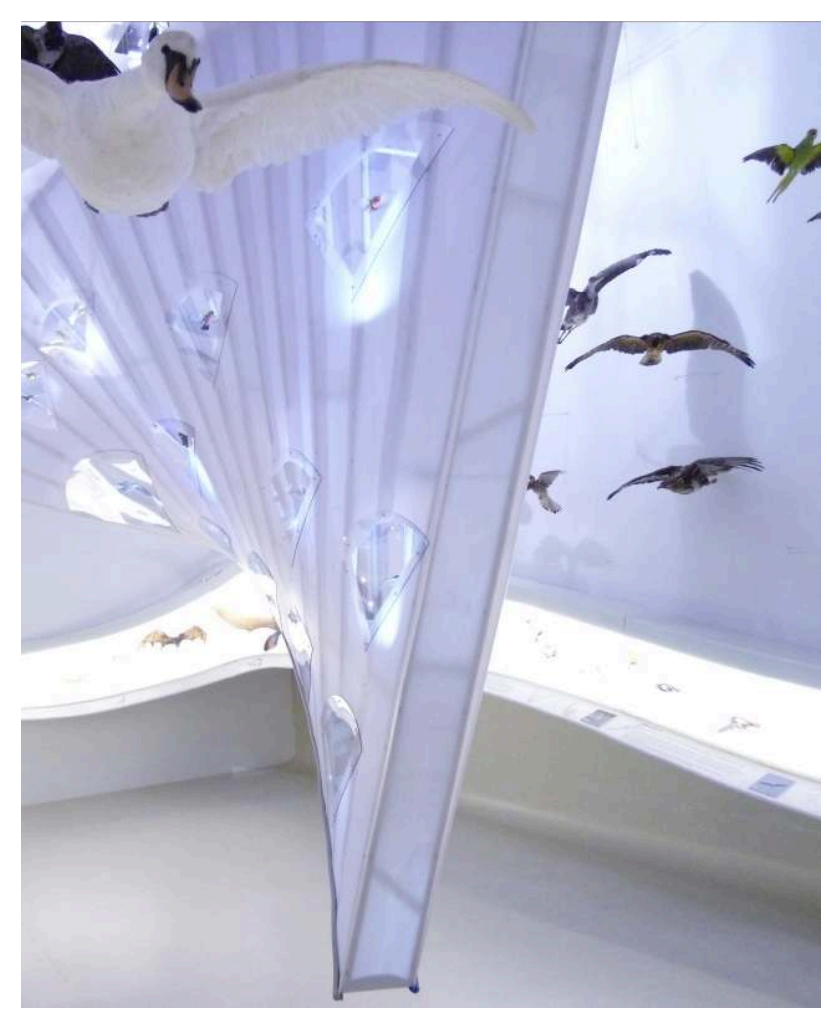

(c) L. Greppi

La profusion d'articles, documentaires, interviews et espaces récemment consacrés à tous les niveaux par les médias démontre combien, dans nos pays, les musées - avec toutes leurs implications sociales, culturelles et économiques - sont devenus un sujet 
d'urgence et de première importance. Tout comme, en parallèle, la croissance exponentielle de l'intérêt du public envers les musées et leurs "manifestations virtuelles » confirme - bien au-delà d'une légitime curiosité et quête de divertissement - le poids et la persévérance de leur présence institutionnelle comme «refuge identitaire » d'une société perdue et dispersée, qui a du mal à retrouver ses points de repère.

Mais, au vu de ces considérations, rouvrir tout simplement les portes des musées comme si de rien n'était ne suffit et ne suffira pas. Car, si le virus revêt un aspect " positif ", c'est qu'il a contribué à dévoiler et à mettre ultérieurement à nu une série de problématiques et de défauts structurels qui se manifestaient déjà depuis longtemps et qu'il est donc absolument temps d'aborder et d'essayer de résoudre. Maintenant ou jamais : car s'il est peut-être trop hasardeux ou ambitieux d'établir que rien ne pourra plus être comme avant, il est par contre encore plus impératif de statuer que rien ne devra plus être comme avant. À partir des musées, de leur rôle dans la société et de leur capacité d'adaptation. De sorte que, sur cet élan, il s'agira, d'une part, de développer de nouvelles stratégies attractives capables de donner à tous les publics, après le déluge de visites virtuelles qui les a submergés tout au long de cette période, l'envie de retourner avec empressement les fréquenter in situ et «à vraie échelle »; d'autre part, il s'agira aussi et surtout de cueillir les instances de changement et de renouveau exprimées par le débat disciplinaire bien avant l'arrivée du Covid-19 : vers la recherche d'un rapport plus synergique et vertueux entre "musées et émotions».

3 Pour bien des musées - et surtout de soi-disant musées " mineurs » - c'est une occasion à ne pas manquer pour transformer de simples questions de survie en opportunités de relance et de repositionnement. Partant avant tout de l'exploitation appropriée de leurs principales ressources : les publics et les collections.

\section{Un musée habité}

4 La première de ces questions prend en considération les aspects liés aux publics. Dans la direction d'un musée plus fortement inclusif et participatif, qui devra tout d'abord être capable de se consacrer et de se focaliser au mieux sur sa propre dimension locale. Là où pour «dimension locale » il ne faut pas voir une diminution de portée ou une réduction d'échelle mais, bien plus, un approfondissement, un agrandissement, un « zoom » dynamique « vers et depuis » le territoire, ses ressources et ses habitants. Là où, de même, les concepts de proximité et de voisinage amènent à une densification des agrégations et à une fortification des relations. Un changement de paradigme et de vision en mesure d'impliquer le contexte territorial dans toutes ses articulations et facettes et de mettre ensemble citoyens, écoles, instituts professionnels et de recherche, productions d'excellence, associations professionnelles, tiers secteur, et autres institutions culturelles jusqu'à jeter les bases d'un véritable système muséal territorial intégré : un système vertueux en mesure de relier la sphère publique à celle du privé ; basé sur un public «réel» de provenance locale ; quantitativement plus limité mais par contre plus stable et fidèle ; moins enclin à la pratique sauvage du « hit and run"; et potentiellement destiné à apporter une grande "valeur ajoutée " précisément parce qu'implicitement plus susceptible d'être motivé, intéressé, impliqué dans les pratiques et la vie de «son » institution. 
"Il s'agit d'aller au-delà de la création de "belles formes" pour mettre en scène une narration efficace et cohérente ». Musées royaux de Turin.

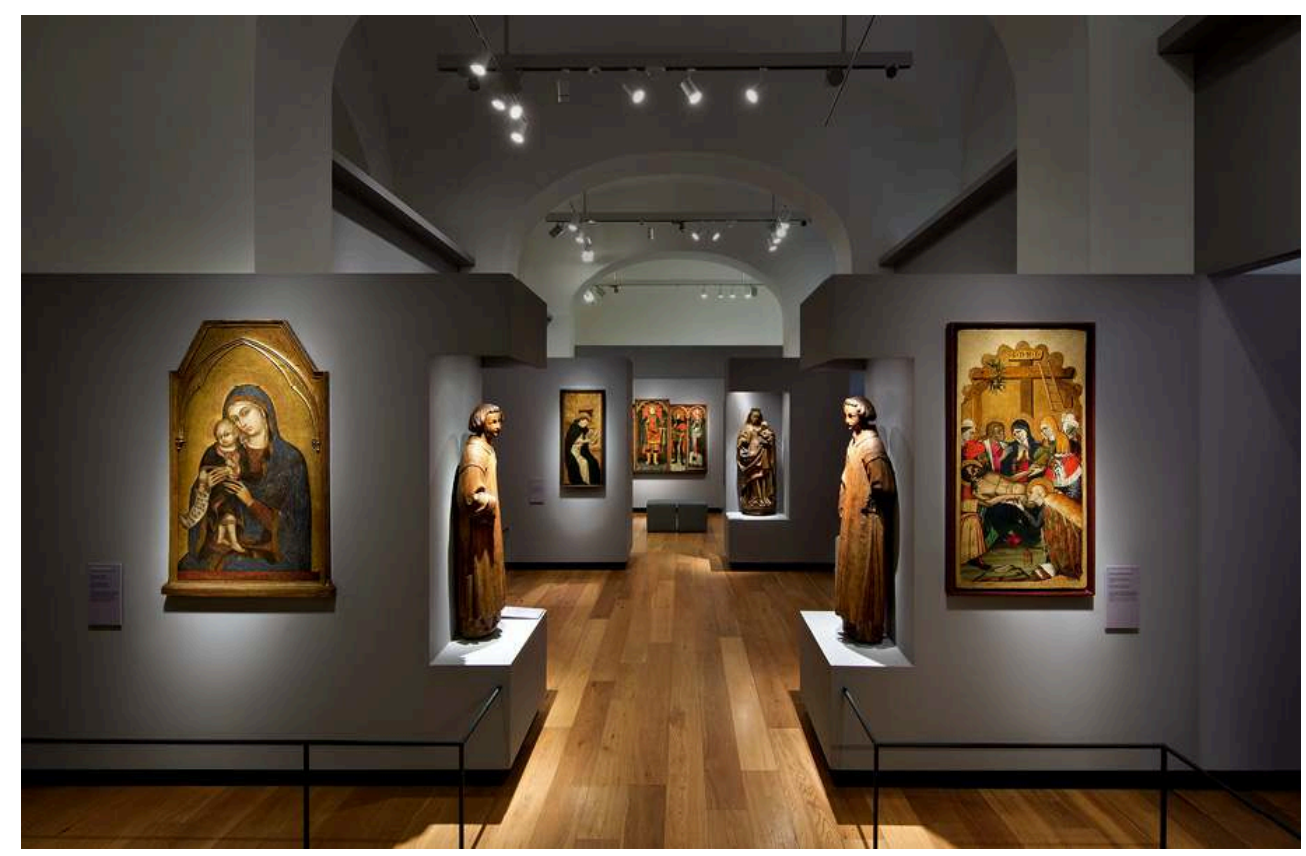

(c) B. Corsico

5 Un musée de proximité capable de mettre en place des formes privilégiées d'accueil, d'attraction, de partage, d'inclusion, de stimuler le sentiment d'appartenance et d'appropriation, d'élaborer de simples systèmes d'abonnement et de billetterie, afin de favoriser la répétition dans le temps d'une expérience de visite qui se fait à chaque fois plus assidue, personnalisée et personnelle, intime et profonde. Jusqu'à transformer les «communities» virtuelles en communautés de personnes en chair et en os, les networks sociaux en réseaux de contacts tangibles, la proximité et le voisinage en une chaîne solide et solidaire et le Musée avec la majuscule en "mon» musée, une institution de façade en une maison démocratique largement partagée : où chacun peut se sentir un peu comme chez soi, transformer son statut de "visiteur» en celui d' " habitant ", conscient du rôle et de la mission qui lui sont confiés, respectueux de ses prérogatives et de ses devoirs. Un musée de fréquentation à la place d'un musée de consommation, où il est facile de revenir, d'approfondir, explorer, penser, s'arrêter, se perdre et se retrouver, de s'offrir une nouvelle dimension de visite sur mesure, de trouver un souffle différent à des coordonnées spatio-temporelles trop longuement compressées : un musée habité, intense, dilaté et plus raréfié, qui s'écoule lentement...

\section{Un musée engagé}

6 La deuxième question prend en compte les aspects liés au capital humain : le capital de personnes (visiteurs, conservateurs, animateurs, professionnels, etc.) que les musées doivent être capables d'attraire, de stimuler et de fidéliser et qui représente, en fin de compte, la part plus inestimable de leur patrimoine, la plus importante de leurs ressources.

7 À cet égard, la dimension largement perçue des musées et de leur statut comme « refuge identitaire » et, en quelque sorte, comme « label de qualité » du territoire offre 
à ceux-ci l'opportunité de mettre le focus sur un engagement socio-culturel plus sensible, attentif et responsable. Ceci dans la direction d'un "musée de citoyenneté ", propulsif et proactif, polyphonique, solidement ancré au centre dynamique de la vie, des instances et des expressions sociales, culturelles et associatives de "sa" communauté d'appartenance : un musée d'écoutes et de dialogues, d'interrogations et de réponses, centrifuge et centripète, qui sort de la carapace de ses limites dimensionnelles acceptant le défi d'ouvrir ses portes, de mettre à disposition sa légitimation de centre de savoirs et de connaissances pour redéfinir de manière plus créative et flexible son propre rôle et celui de ses professionnalités.

$8 \mathrm{Ce}$, au profit d'une nouvelle pratique d'invention et de partage dans laquelle acteurs, metteurs en scène, scénographes, protagonistes principaux et simples spectateurs participent tous ensemble au « bien commun » de leur institution.

Les musées, des « refuges identitaires » d'une société perdue et dispersée en quête de point de repère? Musée d'Histoire naturelle de Venise.

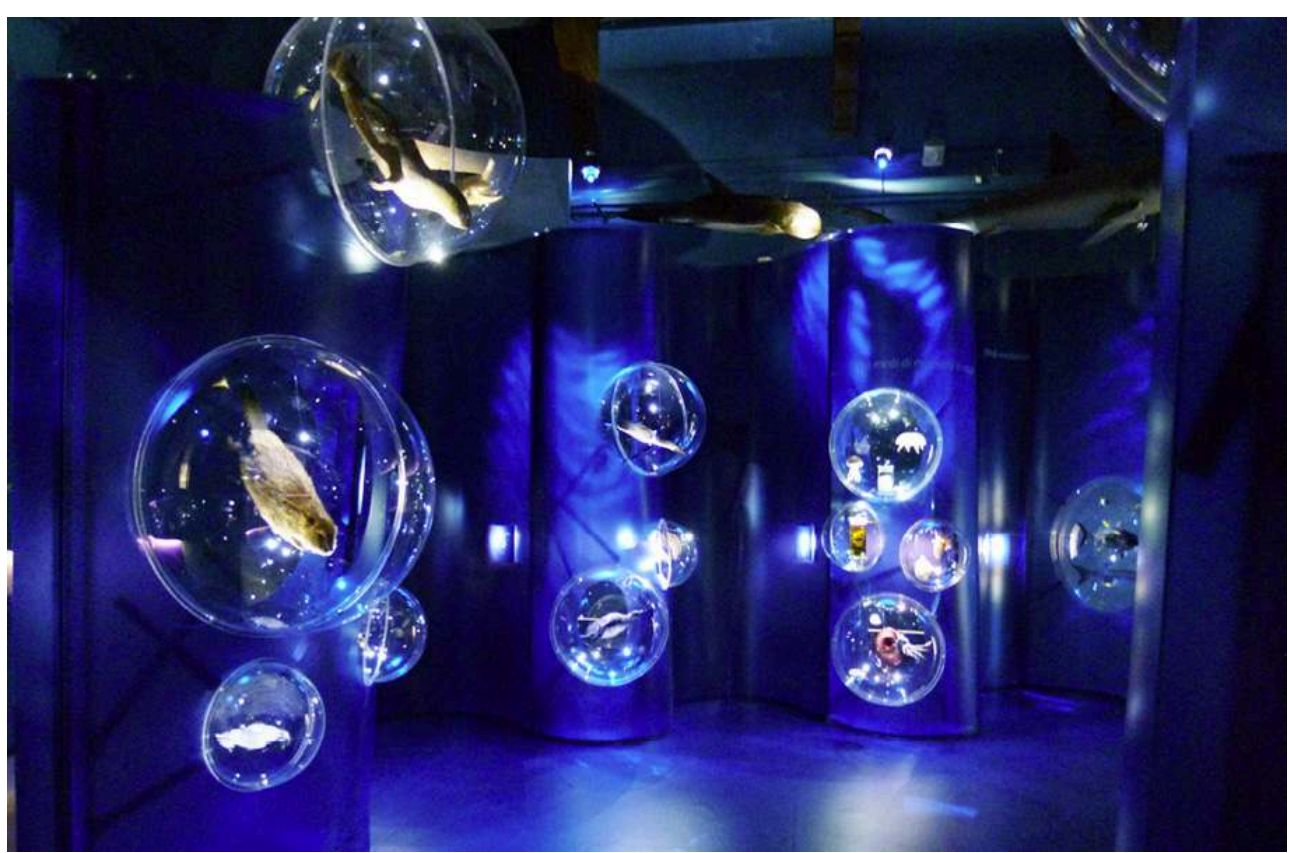

(C) L. Greppi

9 Une nouvelle pratique d'invention qui s'accompagne, d'une part, du repérage et de l'encadrement de profils disciplinaires de "nouvelle génération» (passant de facilitateur, accompagnateur, inventeur, expert en médias sociaux, influenceur à créateur) sur lesquels les musées seront amenés à investir - à l'intérieur tout comme à l'extérieur de leurs murs - dans la perspective de former des équipes de travail concrètement multidisciplinaires, transversales et polyphoniques permettant de lancer et soutenir au mieux les nouveaux processus culturels créatifs qui les attendent dans l'ère de l'après-Covid.

10 Une pratique qui rend d'autre part absolument nécessaire la mise au point d'un " pacte partagé de confiance» entre le musée et son capital humain, au risque de le voir s'amoindrir et de le perdre. Dans ce pacte, le musée doit partir du patrimoine de crédibilité (qui lui vient, d'une part de son statut et, de l'autre, de l'autorité scientifique conquise sur le champ) pour jouer les atouts de la vérité et de la sincérité, de 
l'authenticité, de la concordance entre formes et contenus. Où il s'agit avant tout, et dès le début, d'informer le visiteur, d'afficher la mission, les expériences, les modalités, les conditions de visite du musée, tout comme les règles du jeu pour permettre au visiteur de choisir sa propre démarche et son itinéraire en toute connaissance de cause : en fonction de son temps, de ses intérêts ainsi que de son état d'âme. Et où, au niveau de l'aménagement, il s'agit d'aller bien au-delà de la simple création de "belles formes » ou de « beaux effets ", d'utiliser le design ou les nouvelles technologies comme objectifs et valeurs en soi ou de poursuivre des sujets et des thèmes à la mode, pour mettre en scène une narration efficace et cohérente, au service de la transmission des contenus et de la poétique des émotions.

\section{Réinventer l'exposition virtuelle}

11 La troisième question concerne l'utilisation appropriée de la « dimension virtuelle » des musées : un endroit métaphysique, un espace-temps potentiellement illimité, accessible à tous les publics (également à la part de public plus éloigné qui, du moins dans un premier temps, n'aura pas l'opportunité de se rendre sur place) et qui doit se configurer non seulement comme outil de marketing et ressource de communication culturelle en ligne, mais aussi et surtout, comme instrument de narration et d'émotions.

Car si les nouvelles technologies numériques ont d'une part permis de mettre au point des modalités d'interaction et des systèmes de navigation indubitablement efficaces et intelligents ; de l'autre elles ont par contre quasiment «oublié » de développer des formes appropriées et conséquentes de mise en récit et de narration, se limitant pour la plupart à reproduire virtuellement le parcours d'exposition existant suivant un cliché d'imitation et/ou d'audioguide à distance (nécessairement appauvri et faussé par rapport à la complexité et à l'unicité de la visite « à la vraie échelle »).

Dispositif de l'exposition Le génome humain : ce qui nous rend uniques au musée des Sciences de Trento.

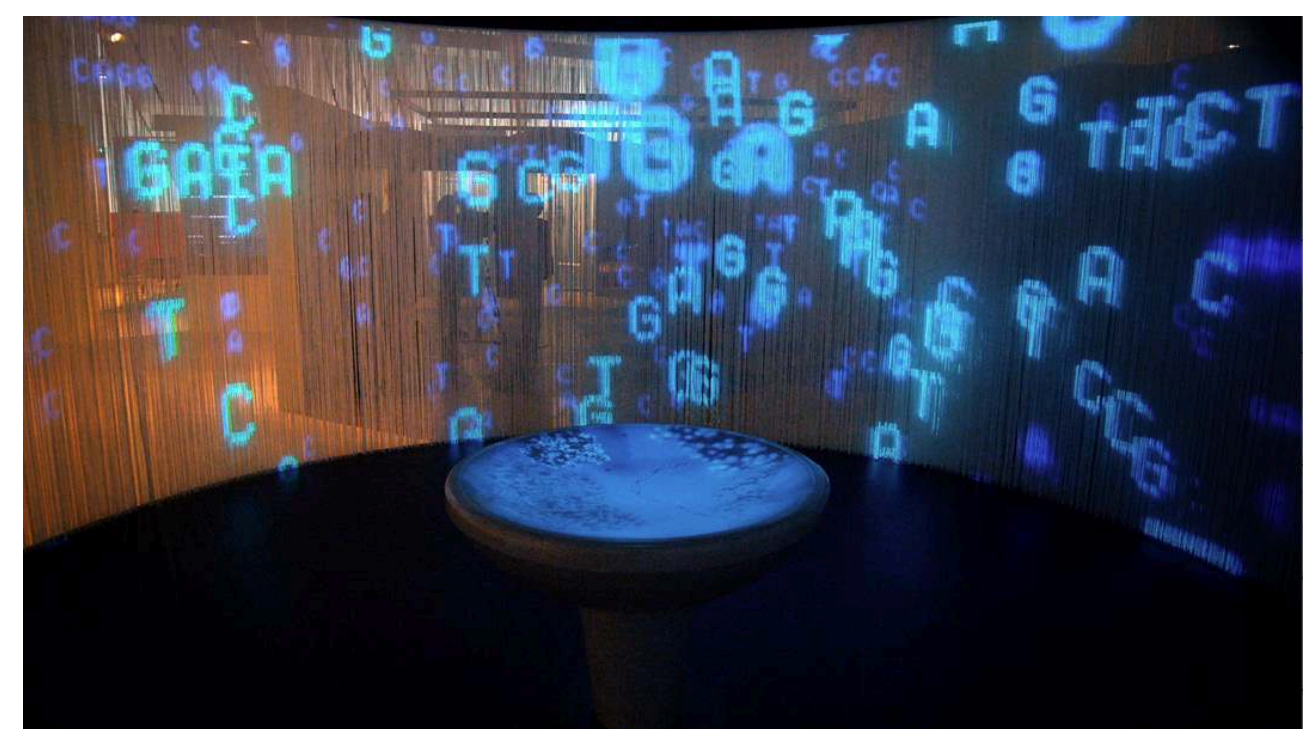

(C) L. Greppi 
13 À partir de ces considérations, travailler sur la dimension virtuelle des musées ne signifie donc pas seulement permettre au public d'avoir accès à une galerie d'images interactives, pouvoir agrandir à démesure les pixels d'une fleur ou d'un tableau (ce que l'artiste en question n'aurait probablement pas voulu), suivre le parcours fléché entre les salles et/ou pratiquer des jeux en ligne. Mais, bien plus : proposer une dimension alternative, différente, inédite, qui - tout en voulant stimuler le navigateur à la visite réelle - constitue elle-même une expérience accomplie de visite parallèle. Une expérience innovante, créative, poétique, qui passe par la mise en scène d'une scénographie virtuelle d'émotions réelles et dans laquelle chaque musée peut aller audelà de ses contraintes dimensionnelles et physiques, peut raconter ses clefs de lecture et ses outils d'interprétation, explorer et sublimer en toute cohérence le récit identitaire de son patrimoine et de son histoire, pour rêver et faire rêver...

\section{Un musée sensible et émotionnel}

14 Le quatrième questionnement concerne le poids et le rôle du patrimoine, matériel et immatériel, qui caractérise chaque musée. Pour un musée de collections - qui ne se concentre plus sur de grandes expositions à consommer et jeter, mais plutôt sur une utilisation dynamique et " créative » de ses collections et de sa structure permanente ceci se concrétise à travers un ensemble intégré de petits et grands gestes ciblés, d'accents, de combinaisons, d'interactions, capables de tirer le meilleur parti du patrimoine muséal existant, de ses archives matérielles et immatérielles, de ses gisements cachés, pour proposer de nouvelles trames et traces narratives, des perspectives et des points de vue inédits, des angulations et des regards à chaque fois différents, dans le contexte d'une dimension de visite continuellement attractive, constamment renouvelée et en évolution pérenne.

15 Là où l'utilisation appropriée de formes innovantes d'aménagements et de contextes scénographiques «émotionnels » représente un moteur d'attraction extrêmement puissant, capable - en toute cohérence - de mettre chaque visiteur à sa « juste place » : qui n'est plus celle traditionnellement subordonnée à la supposée primauté des objets en exposition (autrement, il ne serait pas nécessaire de rouvrir les portes des musées), ni encore moins celle qui le voit situé «au centre» de l'exposition (car pour cela les visites virtuelles suffiraient amplement), mais plutôt une nouvelle place dynamique et dialectique "au cœur» du musée, à l'intérieur de son vivant et de l'expérience de visite, de l'épais réseau de relations entre contenu et conteneur, œuvres, histoires, publics, scénographies, images, imagination et imaginaire qui marquent son unicité, sa non-reproductibilité, son urgence. Soulignant, en extrême synthèse, le degré de sa «préexistence, présence, persistance » dans le temps et l'espace : où chaque musée se structure comme lieu d'un "présent » continuellement en devenir qui témoigne déjà de sa «préexistence » dans le passé tout en posant les bases de sa future "persistance $»$. 
"Une expérience innovante, créative et poétique passe par la mise en scène d'émotions réelles ". Salle " Les fusillés » du musée de la Résistance nationale de Champigny-sur-Marne.

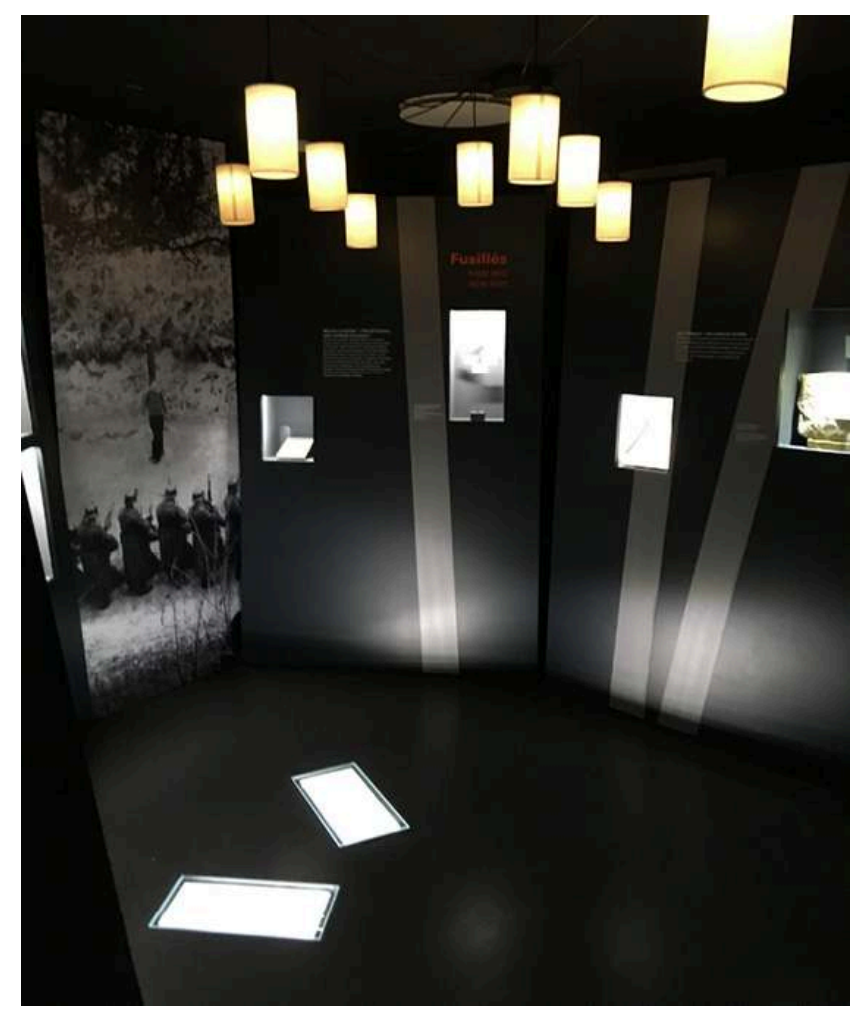

(C) L. Greppi

16 Pour un musée qui se conçoit donc comme lieu privilégié d'émotions : un lieu extraordinaire d'impressions, de sensations, de suggestions, en mesure de communiquer connaissances et informations du plus haut niveau scientifique, disciplinaire et culturel en partant surtout - et avant tout - des émotions, la stupeur, l'émerveillement, de sa capacité de surprendre, émerveiller, étonner, de faire voyager l'imagination, la créativité, la mémoire individuelle et collective, le rêve, la fantaisie, l'imaginaire...

Le tout dans un contexte éthique et social que le Covid-19 aura rendu plus sensible et attentif envers les enjeux du développement durable et de la consommation de la planète : enjeux que les musées de l'ère post-Covid auront le devoir institutionnel, inscrit dans leur génome, et l'opportunité stratégique de prendre en compte ; et qui pourront ainsi devenir un important élément de fidélisation patrimoniale et de débat communautaire, non seulement à travers la numérisation des services et la qualification des offres à distance mais aussi - et surtout - à travers la production de nouvelles consciences. 


\section{RÉSUMÉS}

La crise du Covid-19 a révélé les limites préexistantes de certaines pratiques muséales. La réouverture au public est une occasion sans précédent de proposer des solutions et de repenser les institutions en accord avec leurs ressources, leurs publics et leurs territoires.

INDEX

Mots-clés : expérience de visite ; exposition virtuelle

\section{AUTEUR}

\section{LORENZO GREPPI}

Architecte scénographe

info@lorenzogreppi.com

www.lorenzogreppi.com 